\title{
LoAd Frequency Control for Power System Using Generalized Extended State Observer
}

\author{
Anh-Tuan TRAN ${ }^{1}$, Phong Thanh TRAN ${ }^{1}$, Van Van $H U Y N H^{1, *}$ \\ ${ }^{1}$ Faculty of Electrical \& Electronics Engineering, Ton Duc Thang University, Ho Chi Minh City, \\ Viet Nam \\ *Corresponding Author: Van Van HUYNH (Email: huynhvanvan@tdtu.edu.vn) \\ (Received: 25-Oct-2020; accepted: 13-Jan-2021; published: 31-Mar-2021) \\ DOI: http://dx.doi.org/10.25073/jaec.202151.307
}

\begin{abstract}
This study investigates load frequency control based generalized extended state observer (GESO) for interconnected power system subject to multi-kind of the power plant. First, the mathematical model of the interconnected power system is proposed based on the dynamic model of thermal power plant with reheat turbine and hydro power plant. Second, the GESO is designed to estimate the system states and disturbances. In addition, the problem of unmeasurable of system states in the interconnected power network due to lack of sensor has been solved by using the proposed load frequency control based GESO. The numerical experiments are carried out by using MATLAB/SIMULINK simulation. The simulation results point out that the proposed control approach has capacity to handle the uncertainties and disturbances in the interconnected power system with better transient performances in comparison with the existing control approach. The relevant dynamic models have already been used for the simulation of the physical constraints of governor dead band (GDB) and generation rate constraint (GRC) effect in the power plants. It is evident that the robustness of the suggested controller in terms of stability and effectiveness of the system.
\end{abstract}

\section{Keywords}

Interconnected power system, load frequency control, generalized extended state observer.

\section{Introduction}

In large power systems, load frequency control (LFC) is one of the essential operation problems in electrical power under load and resource variation. Consequently, any changes in frequency not only impact truthfully on the operation of power networks and power system reliability but also lead to an uncertain condition for interconnected power networks. The primary goals of a power network LFC are to keep and maintain the frequency deviation and tie line power exchanges with neighbor control areas at the planned value according to a schedule [1-6].

In a power system, it is not easy to regulate the frequency in power exchange/interchange through tie-lines. Many academic researchers represented the LFC approach of typical power system utilizing different control methods in both traditional and developed techniques that have been applied to resolve the LFC problems of interconnected power networks [7-22].

To resolve problems of frequency deviation occurred by various matched or mismatched uncertainties, adaptive control is one of modern 
control schemes for complex power system included in various load and power control areas [7-10]. LFC control approach for interconnected power networks is introduced to establish in the direct-indirect adaptive fuzzy control strategy [7] which extends and builds up the parameter algorithms and the appropriate adaptive control law. An adaptive model predictive LFC approach in [8] for multi-area power network is used with photovoltaic generation by regrading some nonlinear features. An adaptive LFC controller is suggested [9] by making use of the least square strategy with an internal model control design in structure. In [10], the hypothesis of the suggested adaptive way is constructed by the on-line tuning method of the gain of an integral controller which is applied in the electrosearch optimization. However, the adaptive control schemes above were presented to be complex in control algorithms to deal with the variations of power network parameters subjected to the LFC approaches.

The conventional PID controllers of constant parameters and fixed system structure were investigated to solve the LFC problem for normal operating condition [11-13]. However, the characteristic of power networks is nonlinear system. Therefore, some traditional PID control methods may not have ability to improve the better performance for other operating conditions and to make the best of optimizing the PID parameters and to develop stability performance in power network with the parameter uncertainties and load disturbances.

In the different way of doing the research, sliding mode control (SMC) is not only another way to resolve LFC difficult problems but also a nonlinear control approach to be well known for improving the system performance. In detail, the SMC approach is very sensitive to validate of plant parameters as well as improve effectively in system transient performance. In recent times, there are many the SMC frequency approaches implemented to work out the problems of power networks with parameter uncertainties or load variations [14-17]. The LFC controller is suggested and developed for the interconnected power system to upgrade the system performance using the decentralized SMC [14]. However, the matched and unmatched un- certainties in power networks are not always suitable and satisfied in all of conditions. A novel adaptive SMC approach [15] is designed to the LFC in terms of the parametric derivation the external disturbances. However, there are some existing limitations such as the computation of dynamic model and control design is complex, the state cannot be observed, the response performance and waveform need to improve. In [16], the proposed SMC is applied in the basic control method with the adaptive dynamic programming approach is utilized to improve the extra control signal and to modify the frequency scheme. However, parameter uncertainties due to variable operation point was not considered and the transient performance were not very good as compared previous research. [17] is distributed sliding mode control scheme for optimal LFC to adopt a nonlinear model of a multi-area power system, including dynamic of voltage and dynamics of second order turbinegovernor. However, the suggested strategy is complicated and difficult for practical implementation. The parameter uncertainties of power generation were not discussed.

From above control researches such as adaptive control, PID control and SMC control, the results are achieved under assumption that all state variables of power system can to be measurable and willingly available for system feedback. In practical application, not all state variables are measurable for system feedbacks, and then we must compute and estimate the state variables that cannot measure in the system. The proposed approach of estimating state variables is often used in observation [18-22], [2629]. These schemes can modify and improve the unknown upper bounds of matched and mismatch uncertainties. It not only obtains the system state trajectories accomplishment but also satisfies in parameters of the system state errors. The achievement of results is correlated to LFC's of power networks in various control techniques [18-22], [26-29]. However, there are still some limitations of the above approaches such as the disturbances are not truncated from the output points in steady state and the gains of controller are not set to be really high to reduce disturbances of unknown boundaries and even, the parameter uncertainties or load variations 
of power system are not considered in some proposed controllers. The GESO scheme based on the non-linear SMC controller are combined to investigate the frequency variation problem and to estimate the disturbance in interconnected power network [30]. However, the performance of power system is not always satisfied under required conditions in the settling time, transient frequency variations considering GDB and GRC effect. In detail, GRC is normally considered by adding a limiter and a hysteresis pattern to the governor-turbine system model. It is essential to take into consideration the practical constraints and natural conditions such as physical constraint of generation rate constraint (GRC). The GRC has negative affect on the power network performance due to its nonlinearity nature. The GRC denotes practical constraint on the ratio of the variation in the generating power due to physical drawbacks. Governor dead band (GDB) is principle for power network frequency control in the presence of disturbances. GDB has a definite outline as the total magnitude of a continued speed change that there is no resulting variation in valve position. An observer-based control scheme is offered for LFC scheme against cyberattack uncertainties [31]. However, to test the effective response of the proposed controller of power networks, the GDB and GRC effect are not considered in the power plant.

The process of being mentally stimulated to do the control approaches is to eliminate and improve perturbations by feedback control instead of feedforward compensation control which uses the disturbance estimations to cancel out the affections in real time manner. The suggested control approach is one way of approaches for estimating and compensating the system disturbances. This scheme proves the powerful and robustness to again matched uncertainties. The contributions of the proposed GESO in this paper are as follows:

- GESO is designed to estimate the unmeasurable system state variables and the load uncertainties in the complex power system. The proposed scheme of making power system is not only secure or stable but also useful to solve the satisfactory performance with uncertainties.
- The generalized extended observer approach improves the system dynamic response to fast response in setting time and to reduce over or undershoots in power network with the dynamic model of thermal power plant with reheat turbine and hydro power plant.

- The simulation result with various cases indicates the effectively and robustness of generalized extended controller by considering parametric uncertainties in power networks.

- The report of simulation results is used to compare with the cases of considering and without considering the GDB and GRC nonlinearity effects on power network.

The paper is outlined as follows: section II shows the mathematical model of power network. The generalized extended state observer for multi-area power systems is designed in section III. Section IV represents stability analysis of power system and control scheme design of power system, the following session by simulation results in section $\mathrm{V}$.

\section{Mathematical model of power system}

The dynamic models of power systems are generally nonlinear. The block chart of power system is presented in Fig. 1, Fig. 2 and Fig. 3. The power system includes two types of the plant as thermal power plant with reheat turbine and hydro power plant with mechanical hydraulic governor connected through tie-line power.

\subsection{The thermal power plant with reheat turbine model}

A thermal power plant is an electric power station to convert heat energy to electric power. Reheat turbine is a part of thermal power plants in power network in Fig. 2.

The frequency dynamic behavior of area $i^{\text {th }}$ details in this section which can use in the fol- 


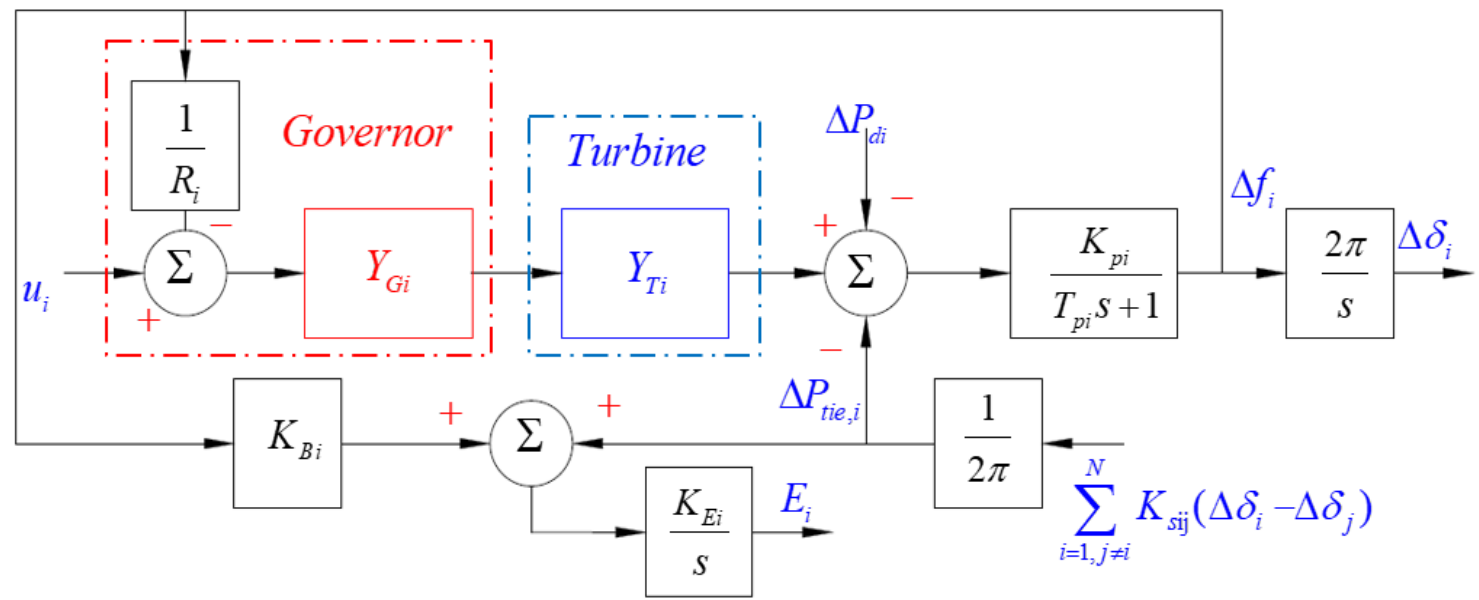

Fig. 1: The block chart of complex power network.

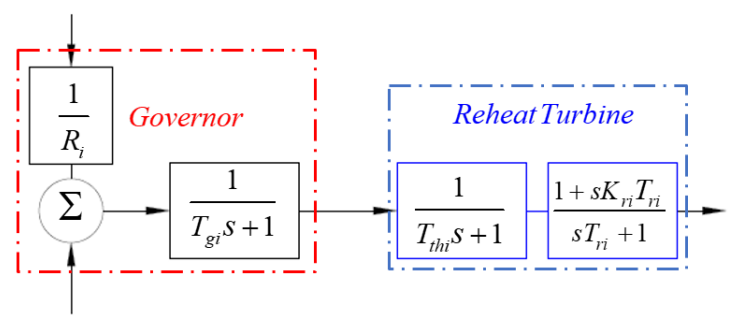

Fig. 2: The block chart of $i^{t h}$ area with reheat turbine.

lowing differential equations:

$$
\begin{aligned}
\Delta \dot{f}_{i}(t)= & -\frac{1}{T_{p i}} \Delta f_{i}(t)+\frac{K_{P i}}{T_{p i}} \Delta P_{m i}(t) \\
& -\frac{K_{P i}}{2 \pi T_{P i}} \sum_{i=1, j \neq i}^{N} K_{s i j}\left[\Delta \delta_{i}(t)-\Delta \delta_{j}(t)\right] \\
& -\frac{K_{P i}}{T_{p i}} \Delta P_{d i}(t)
\end{aligned}
$$

$\Delta \dot{P}_{m i}(t)=\left(\frac{1}{T_{r i}}-\frac{K_{r i}}{T_{t h i}}\right) \Delta P_{t h i}(t)+\frac{K_{r i}}{T_{t h i}} \Delta P_{v i}(t)$

$$
-\frac{1}{T_{r i}} \Delta P_{m i}(t)
$$

$$
\begin{aligned}
\Delta \dot{P}_{t h i}(t)= & \frac{1}{T_{t h i}} \Delta P_{v i}(t)-\frac{1}{T_{t h i}} \Delta P_{t h i}(t) \\
\Delta \dot{P}_{v i}(t)= & -\frac{1}{T_{g i} R_{i}} \Delta f_{i}(t)-\frac{1}{T_{g i}} \Delta P_{v i}(t) \\
& +\frac{1}{T_{g i}} u_{i}(t)
\end{aligned}
$$

$$
\begin{aligned}
& \Delta \dot{E}_{i}(t)= K_{B i} K_{E i} \Delta f_{i}(t) \\
&+ \frac{K_{E i}}{2 \pi} K_{s i j}\left[\Delta \delta_{i}(t)-\Delta \delta_{j}(t)\right] \\
& \Delta \dot{\delta}_{i}(t)=2 \pi \Delta f_{i}(t)
\end{aligned}
$$

The variables of interconnected power system are the frequency deviation, power output, governor valve position, integral control and rotor angle variation as following as bellow:

$$
x_{i}(t)=\left[\begin{array}{c}
\Delta f_{i}(t) \\
\Delta P_{m i}(t) \\
\Delta P_{t h i}(t) \\
\Delta P_{v i}(t) \\
\Delta E_{i}(t) \\
\Delta \delta_{i}(t)
\end{array}\right]
$$

where $i=1,2, . ., N$ and $N$ is the area numbers.

The interconnected power network with thermal power plant described by Fig. 1 and Fig. 2 , which can be written and expressed in statespace representation below:

$$
\begin{aligned}
\dot{x}_{i}(t)= & A_{i} x_{i}(t)+B_{i} u_{i}(t) \\
& +\sum_{i=1, i \neq j}^{N} H_{i j} x_{j}+\Gamma_{i} \Omega_{i}(t) \\
y_{i}(t)= & C_{i} x_{i}(t)
\end{aligned}
$$


The definition of following matrices is as given in the following:

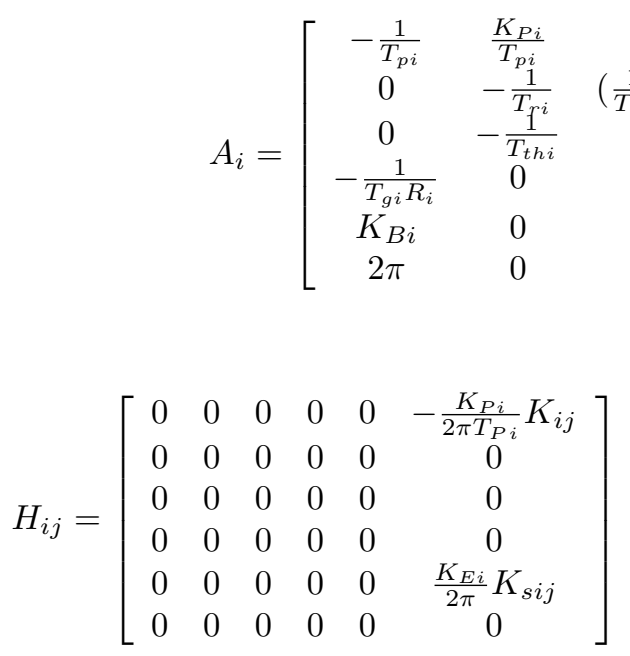

$$
\begin{aligned}
& B_{i}=\left[\begin{array}{llllll}
0 & 0 & 0 & \frac{1}{T_{g i}} & 0 & 0
\end{array}\right]^{T} \\
& \Gamma_{i}=\left[\begin{array}{llllll}
-\frac{K_{P i}}{T_{p i}} & 0 & 0 & 0 & 0 & 0
\end{array}\right]^{T} \\
& \left.\begin{array}{cccc}
0 & 0 & 0 & -\frac{K_{P i}}{2 \pi T_{P i}} K_{i j} \\
\left.\frac{1}{T_{r i}}-\frac{K_{r i}}{T_{t h i}}\right) & \frac{K_{r i}}{T_{t h i}} & 0 & 0 \\
\frac{1}{T_{t h i}} & 0 & 0 & 0 \\
0 & -\frac{1}{T_{g i}} & 0 & 0 \\
0 & 0 & 0 & \frac{K_{E i}}{2 \pi} K_{s i j} \\
0 & 0 & 0 & 0
\end{array}\right]
\end{aligned}
$$

where $x_{i}(t) \in R^{n_{i}}$ is state vector of the $i^{t h}$ area, $x_{j}(t) \in R^{n_{i}}$ is state vector of the $j^{t h}$ area, $u_{i}(t) \in R^{m_{i}}$ is the control vector, $\Omega_{i}(t) \in R^{m}$ is the vector of the load disturbance, $y_{i}(t)$ is the output signal of the $i^{\text {th }}$ area, $A_{i}$ is the variable matrix of the power system of the $i^{t h}$ area, $B_{i}$ is the output matrix of the $i^{\text {th }}$ area, $H_{i j}$ is the power flow between two-area, $C_{i}$ is the output matrix of the $i^{\text {th }}$ area, $\Gamma_{i}$ is the disturbance matrix of the $i^{t h}$ area, $\Delta \dot{f}_{i}(t)$ is the frequency deviation of the $i^{t h}$ area, $\Delta P_{m i}(t)$ is the mechanics power variation of the turbine of the $i^{\text {th }}$ area and $\Delta \dot{\delta}_{i}(t)$ is the rotor angle deviation of the $i^{t h}$ area.

\subsection{The hydro power plant with hydro turbine}

The hydroelectric power plant is the most common type of power system in Fig. 3. The function of a hydraulic turbine is to convert the energy of the flowing water into mechanical energy and this mechanical energy is converted from hydro-electric generator to electricity.

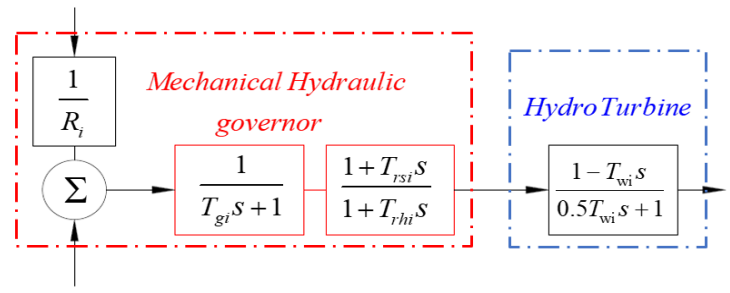

Fig. 3: The block chart of $i^{t h}$ area with hydro turbine.

$$
\begin{aligned}
\Delta \dot{f}_{i}(t)= & -\frac{1}{T_{p i}} \Delta f_{i}(t)+\frac{K_{p i}}{T_{p i}} \Delta P_{m i}(t) \\
& -\frac{K_{p i}}{T_{p i}} \Delta P_{d i}(t) \\
& -\frac{K_{P i}}{2 \pi T_{P i}} K_{s i j}\left[\Delta \delta_{i}(t)-\Delta \delta_{j}(t)\right]
\end{aligned}
$$

$$
\begin{aligned}
\Delta P_{m i}(t)= & \frac{2 T_{r s i}}{T_{r h i} T_{g i} R_{i}} \Delta f_{i}(t) \\
& +\left(\frac{2}{T_{w i}}+\frac{2}{T_{r h i}}\right) \Delta P_{v i}(t) \\
& -\frac{2}{T_{w i}} \Delta P_{m i}(t)-\frac{2}{T_{w i}} \Delta P_{m i}(t) \\
& +\left(\frac{2 T_{r s i}}{T_{r h i} T_{g i}}-\frac{2}{T_{r h i}}\right) \Delta P_{g i}(t) \\
& -\frac{2 T_{r s i}}{T_{r h i} T_{g i}} u_{i}(t)
\end{aligned}
$$

$$
\begin{aligned}
\Delta P_{v i}(t)= & \frac{-T_{r s i}}{T_{r h i} T_{g i} R_{i}} \Delta f_{i}(t)+\frac{-1}{T_{r h i}} \Delta P_{v i}(t) \\
& +\left(\frac{1}{T_{r h i}}-\frac{T_{r s i}}{T_{r h i} T_{g i}}\right) \Delta P_{g i}(t) \\
& +\frac{T_{r s i}}{T_{r h i} T_{g i}} u_{i}(t)
\end{aligned}
$$




$$
\begin{aligned}
\Delta \dot{P}_{g i}(t)= & -\frac{1}{T_{g i} R_{i}} \Delta f_{i}(t)-\frac{1}{T_{g i}} \Delta P_{g i}(t) \\
& +\frac{1}{T_{g i}} u_{i}(t) \\
& \Delta \dot{\delta}_{i}(t)=2 \pi \Delta f_{i}(t)
\end{aligned}
$$

The variables of interconnected power system are the frequency deviation, power output, governor valve position, integral control and rotor angle variation as following as bellow:

$$
x_{i}(t)=\left[\begin{array}{c}
\Delta f_{i}(t) \\
\Delta P_{m i}(t) \\
\Delta P_{v i}(t) \\
\Delta P_{g i}(t) \\
\Delta E_{i}(t) \\
\Delta \delta_{i}(t)
\end{array}\right]
$$

where $i=1,2, . ., N$ and $N$ is the area numbers.

The interconnected power system with hydro power plant described by Fig. 1 and Fig. 3, which can be written and expressed in statespace representation below:

$$
\begin{aligned}
\dot{x}_{i}(t)= & A_{i} x_{i}(t)+B_{i} u_{i}(t) \\
& +\sum_{i=1, i \neq j}^{N} H_{i j} x_{j}+\Gamma_{i} \Omega_{i}(t) \\
y_{i}(t)= & C_{i} x_{i}(t)
\end{aligned}
$$

The definition of following matrices is as given in:

$$
\begin{gathered}
B_{i}=\left[\begin{array}{cccccc}
0 & -\frac{2 T_{r s i}}{T_{r h i} T_{g i}} & \frac{T_{r s i}}{T_{r h i} T_{g i}} & \frac{1}{T_{g i}} & 0 & 0
\end{array}\right]^{T} \\
\Gamma_{i}=\left[\begin{array}{ccccccc}
-\frac{K_{P i}}{T_{p i}} & 0 & 0 & 0 & 0 & 0
\end{array}\right]^{T} \\
H_{i j}=\left[\begin{array}{cccccc}
0 & 0 & 0 & 0 & 0 & -\frac{K_{P i} i}{2 \pi T_{P i}} K_{s i j} \\
0 & 0 & 0 & 0 & 0 & 0 \\
0 & 0 & 0 & 0 & 0 & 0 \\
0 & 0 & 0 & 0 & 0 & 0 \\
0 & 0 & 0 & 0 & 0 & \frac{K_{E i}}{2 \pi} K_{s i j} \\
0 & 0 & 0 & 0 & 0 & 0
\end{array}\right]
\end{gathered}
$$

$$
A_{i}=\left[\begin{array}{cccccc}
-\frac{1}{T_{p i}} & \frac{K_{p i}}{T_{p i}} & 0 & 0 & 0 & -\frac{K_{P i}}{2 \pi T_{P i}} K_{s i j} \\
\frac{2 T_{r s i}}{T_{r h i} T_{g i} R_{i}} & -\frac{2}{T_{w i}} & \left(\frac{2}{T_{w i}}+\frac{2}{T_{r h i}}\right) & \left(\frac{2 T_{r s i}}{T_{r h i} T_{g i}}-\frac{2}{T_{r h i}}\right) & 0 & 0 \\
\frac{-T_{r s i}}{T_{r h i} T_{g i} R_{i}} & 0 & \frac{1}{T_{r h i}} & \left(\frac{1}{T_{r h i}}-\frac{T_{r s i}}{T_{r h i} T_{g i}}\right) & 0 & 0 \\
-\frac{1}{T_{g i} R_{i}} & 0 & 0 & -\frac{1}{T_{g i}} & 0 & 0 \\
K_{B i} & 0 & 0 & 0 & 0 & \frac{K_{E i}}{2 \pi} K_{s i j} \\
2 \pi & 0 & 0 & 0 & 0 & 0
\end{array}\right]
$$

\section{Generalized extended state observer design}

In practical application, not all state variables are measurable for system feedbacks, and then we must compute and estimate the state variables that cannot measure for the parametric uncertainties in power system. Therefore, states observer performs the function by estimating the state variables of the systems typically the output and control variable. State observers can be designed and applied only when the observability of required condition is satisfied. The GESO is recommended for using in this system because it is more sensitive with changes in parameters of load disturbance variations. To estimate state variables in the power system included both variables and load disturbances, GESO is defined as following bellow:

$$
\left[x_{i}\right]_{n_{i}+1}(t)=\Omega_{i}(t)
$$

and

$$
\gamma_{i}(t)=\frac{\Omega_{i}(t)}{d t}
$$

Then, the system in equation can be presented below:

$$
\begin{aligned}
\dot{\bar{x}}_{i}(t)= & \bar{A}_{i} \bar{x}_{i}(t)+\bar{B}_{i} u_{i}(t) \\
& +\sum_{i=1, i \neq j}^{N} \bar{H}_{i j} x_{j}+F_{i} \gamma_{i}(t) \\
\bar{y}_{i}(t)= & \bar{C}_{i} \bar{x}_{i}(t)
\end{aligned}
$$

where

$$
\bar{x}_{i}(t)=\left[\begin{array}{l}
{\left[x_{i}\right]_{n_{i} \times 1}} \\
{\left[\left(x_{i}\right)_{n_{i}+1}\right]_{n_{-} \times 1}}
\end{array}\right]_{\left(n_{i}+n_{i}\right) \times 1}
$$




$$
\begin{gathered}
\bar{A}_{i}=\left[\begin{array}{cc}
{\left[A_{i}\right]_{n_{i} \times n_{i}}} & I_{n_{i} \times n_{i}} \\
0_{n_{i} \times n_{i}} & 0_{n_{i} \times n_{i}}
\end{array}\right]_{\left(n_{i}+n_{i}\right) \times\left(n_{i}+n_{i}\right)} \\
\bar{H}_{i j}=\left[\begin{array}{cc}
{\left[H_{i j}\right]_{n_{i} \times n_{i}}} & 0_{n_{i} \times n_{i}} \\
0_{n_{i} \times n_{i}} & 0_{n_{i} \times n_{i}}
\end{array}\right]_{\left(n_{i}+n_{i}\right) \times\left(n_{i}+n_{i}\right)} \\
\bar{B}_{i}=\left[\begin{array}{c}
{\left[B_{i}\right]_{n_{i} \times p_{i}}} \\
0_{n_{i} \times p_{i}}
\end{array}\right]_{\left(n_{i}+n_{i}\right) \times p_{i}} \\
F_{i}=\left[\begin{array}{c}
0_{n_{i} \times r_{i}} \\
I_{n_{i} \times r_{i}}
\end{array}\right]_{\left(n_{i}+n_{i}\right) \times r_{i}} \\
\bar{C}_{i}=\left[\begin{array}{c}
{\left[C_{i}\right]_{m_{i} \times n_{i}}} \\
0_{m_{i} \times n_{i}}
\end{array}\right]_{m_{i} \times\left(n_{i}+n_{i}\right)}
\end{gathered}
$$

Assumption 1: $\left(A_{i}, B_{i}\right)$ is controllable and $\left(\bar{A}_{i}, \bar{C}_{i}\right)$ is observable.

With regards to the state observers discussed, we will apply the notation $\hat{\bar{x}}_{i}$ to indicate the vector observer state. The vector $\hat{\bar{x}}_{i}$ of the observed state is used and applied in the state feedback to initiate the desired and required control vector.

If we call the state $\bar{x}_{i}$ is approximated to state $\hat{\bar{x}}_{i}$, the dynamical model in (17):

$$
\begin{aligned}
\dot{\overline{\hat{x}}}_{i}(t)= & \bar{A}_{i} \hat{\bar{x}}_{i}(t)+\bar{B}_{i} u_{i}(t) \\
& +\sum_{i=1, i \neq j}^{N} \bar{H}_{i j} \hat{\bar{x}}_{j}+L_{i}\left(\bar{y}_{i}(t)-\hat{\bar{y}}_{i}(t)\right) \\
\dot{\bar{y}}_{i}(t)= & \bar{C}_{i} \hat{\bar{x}}_{i}(t)
\end{aligned}
$$

where, $\dot{\bar{y}}_{i}(t)$ is the estimator state of the output variables and matrix $L_{i}$ is the observer gain, which can be designed through to place any desired eigenvalues in the left half s-plane of $\left(\bar{A}_{i}-L_{i} \bar{C}_{i}\right)$.

The control input is chosen as following as (18):

$$
u_{i}(t)=K_{x i} x_{i}(t)+K_{d i} \hat{\Omega}_{i}(t)
$$

or

$$
u_{i}(t)=K_{x i} \hat{x}_{i}(t)+K_{-d i} \hat{\Omega}_{i}(t)
$$

where $\hat{\Omega}_{i}(t)=\left[\hat{x}_{i}\right]_{n_{i}+1}$

From Eqs. (18) and (19), we can rewrite:

$$
u_{i}(t)=K_{i} \hat{\bar{x}}_{i}(t)=\left[\begin{array}{ll}
K_{x i} & K_{d i}
\end{array}\right] \hat{\bar{x}}_{i}(t)
$$

where, $K_{x i}$ is the feedback control gain to be chosen so that the eigenvalues of $\left(A_{i}-B_{i} K_{x i}\right)$ lie in specific locations in the left-half s-plane and the lumped uncertainty compensation gain $K_{d i}$ is designed:

$$
\begin{aligned}
& K_{d i} \\
& =\left[C_{i}\left(A_{i}-B_{i} K_{x i}\right)^{-1} B_{i}\right]^{-1} C_{i}\left(A_{i}-B_{i} K_{x i}\right)^{-1} \Gamma_{i}
\end{aligned}
$$

To solve the disturbance compensation gain: The disturbance compensation gain in (21) is no longer available since $C_{i}\left(A_{i}-B_{i} K_{x i}\right)^{-1} B_{i}$ is probably non-invertible or even not a square matrix. In a case in point, it can be proved that an alternative but more typical condition.

$$
\begin{aligned}
& {\left[C_{i}\left(A_{i}-B_{i} K_{x i}\right)^{-1} B_{i}\right]^{-1} K_{d i}} \\
& =C_{i}\left(A_{i}-B_{i} K_{x i}\right)^{-1} \Gamma_{i}
\end{aligned}
$$

It must be gratified to assure the viability of the proposed control scheme. The gain $K_{d i}$ can be resolved from (22) if the following rank condition holds:

$$
\begin{gathered}
\operatorname{rank}\left[C_{i}\left(A_{i}-B_{i} K_{x i}\right)^{-1} B_{i}\right]^{-1} \\
=\operatorname{rank}\left[C_{i}\left(A_{i}-B_{i} K_{x i}\right)^{-1} B_{i}\right]^{-1}, \\
{\left[C_{i}\left(A_{i}-B_{i} K_{x i}\right)^{-1} \Gamma_{i}\right]}
\end{gathered}
$$

The simple configuration of the suggested GESO is presented in Fig. 4. It shows the uncertainties which can be designed and eliminated from the output channel in steady state by this control law.

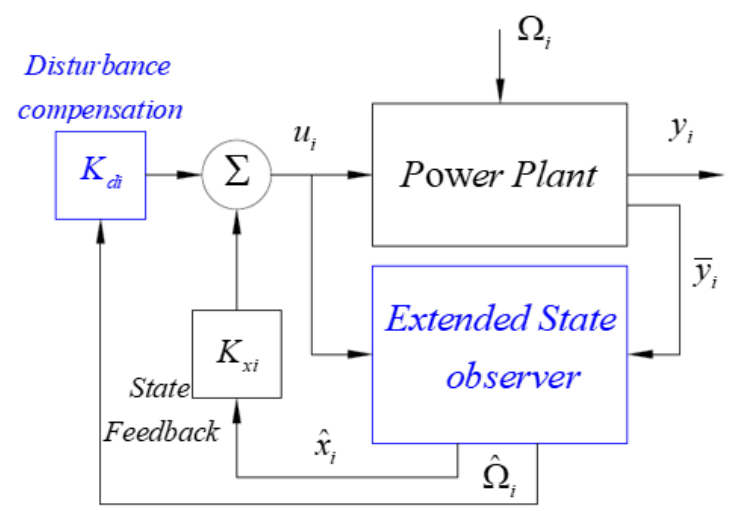

Fig. 4: The configuration of the proposed GESO [2325], [30-31]. 


\section{Stability of power system}

The stability analysis of the multi-area power system as represented in Fig. 1 is performed in this part. The suggested control approach goals at the fundamental boundedness of all the power system signals.

Assumption 2: The lumped disturbances have to the satisfaction of the following conditions.

They get constant value in steady state of system, i.e.; $\lim _{t \rightarrow \infty} \Omega_{i}(t)=\zeta_{i}$ and $\lim _{t \rightarrow \infty} \gamma_{i}(t)=$ $\lim _{t \rightarrow \infty} \dot{\Omega}_{i}(t)=0$.

The state and disturbance estimation errors are defined as:

$$
e_{x i}(t)=\hat{x}_{i}(t)-x_{i}(t)
$$

and

$$
e_{d i}(t)=\hat{\Omega}_{i}(t)-\Omega_{i}(t)
$$

where: $\hat{\Omega}_{i}(t)=\left[\hat{x}_{i}\right]_{n_{i}+1}$ is presented the estimation of the system uncertainties.

Combine (16) and the estimation error of state observers $e_{i}(t)=\bar{x}_{i}(t)-\hat{\bar{x}}_{i}(t)$ can be revised by:

$$
\begin{aligned}
\dot{e}_{i}(t) & =\bar{A}_{i} e_{i}(t)-L_{i}\left(\bar{y}_{i}(t)-\hat{\bar{y}}_{i}(t)\right)+F_{i} \gamma_{i}(t) \\
& =\left(\bar{A}_{i}-L_{i} \bar{C}_{i}\right) e_{i}(t)+F_{i} \gamma_{i}(t)
\end{aligned}
$$

Denote $F_{i} \gamma_{i}(t)$ by $u_{i}(t)$ and use final-value theorem, we can be obtained:

$$
\begin{aligned}
& \lim _{t \rightarrow \infty} e_{i}(t) \\
& =\lim _{t \rightarrow \infty} s\left(s I-\left(\bar{A}_{i}-L_{i} \bar{C}_{i}\right)\right)^{-1} U_{i}(s) \\
& =\lim _{t \rightarrow \infty}\left(s I-\left(\bar{A}_{i}-L_{i} \bar{C}_{i}\right)\right)^{-1} \lim _{s \rightarrow \infty} s U_{i}(s) \\
& =\lim _{s \rightarrow \infty}\left(s I-\left(\bar{A}_{i}-L_{i} \bar{C}_{i}\right)\right)^{-1} \lim _{t \rightarrow \infty} u_{i}(t)
\end{aligned}
$$

Since $\lim _{s \rightarrow \infty}\left(s I-\left(\bar{A}_{i}-L_{i} \bar{C}_{i}\right)\right)^{-1}$ is bounded and $\lim _{t \rightarrow \infty} u_{i}(t)=0$

So, the estimation error of state observers is: $e_{i}(t)=\bar{x}_{i}(t)-\hat{\bar{x}}_{i}(t)$ is asymptotically stable.

Remark 1: By applying the control design to the estimation of the lumped uncertainty and the parameters of system states if the system states are not measurable. So, the proposed control law will be designed as in [23-25].

Remark 2: It is recognized that the lumped uncertainty cannot be reduced completely and totally from the state equation no matter what controller was designed. In this strategy, one of the most recent achievable aims is simply to truncate the disturbances at the output point in steady state by applying of the proposed control law. Therefore, the limitations by other control approaches in the previous papers [18-22] have been resolved.

\section{Simulations and results}

To test the efficiency and robustness of the proposed control strategy, the various cases in two simulations are implemented to prove the performance of GESO controller in estimating of states and avoiding the effect of matched uncertainties with external disturbance. It also reduces effect of the governor dead band (GDB) and generation rate constraint (GRC) in the power plants. These parameters of power network are presented in Table 1 . The simulation results are utilized to compare with previous proposed control scheme in [5-6], [15] using MATLAB/SIMULINK.

Tab. 1: The parameters of interconnected multi-area power system [5-6], [15].

\begin{tabular}{|l|l|l|}
\hline No. & Parameters & Value \\
\hline 1 & $T_{g i}$ & 0.08 \\
\hline 2 & $T_{t h i}$ & 0.3 \\
\hline 3 & $K_{r i}$ & 0.5 \\
\hline 4 & $T_{r i}$ & 10 \\
\hline 5 & $T_{r s i}$ & 5 \\
\hline 6 & $T_{r h i}$ & 28.75 \\
\hline 7 & $T_{W i}$ & 0.3 \\
\hline 8 & $R_{i}$ & 2.4 \\
\hline 9 & $T_{p i}$ & 20 \\
\hline 10 & $K_{p i}$ & 120 \\
\hline 11 & $K_{b i}$ & 0.425 \\
\hline 12 & $a_{\mathrm{ij}}$ & -1 \\
\hline 13 & $2 \pi K_{s i j}$ & 0.215 \\
\hline
\end{tabular}

We can issue the estimating value to find the real value. The observer gain is designed depend on the pole $\rho$ with the eigenvalue $\bar{A}_{i}-L_{i} \bar{C}_{i}$ of lied in the desired locations in the left-half s-plane. 


\section{Simulation 1:}

Case 1. In this case, we apply proposed controller for interconnected power network with only thermal power plant in both stations in Fig. 1 and Fig. 2.

To combine between system matrix of thermal power plant and parameter values in Table 1, the matrix values of the power network are calculated as:

$$
\begin{gathered}
A_{1}=\left[\begin{array}{cccccc}
-0.05 & 6 & 0 & 0 & 0 & -0.0327 \\
0 & -0.1 & -15667 & 1,6667 & 0 & 0 \\
0 & -3.3333 & 3.3333 & 0 & 0 & 0 \\
-5.2083 & 0 & 0 & -12.5 & 0 & 0 \\
0.425 & 0 & 0 & 0 & 0 & 0.0054 \\
6.2832 & 0 & 0 & 0 & 0 & 0
\end{array}\right] \\
B_{1}=\left[\begin{array}{lllllc}
0 & 0 & 0 & 12.5 & 0 & 0
\end{array}\right]^{T} \\
\Gamma_{1}=\left[\begin{array}{llllll}
-6 & 0 & 0 & 0 & 0 & 0
\end{array}\right]^{T}
\end{gathered}
$$

Feedback control gain in this case can be designed as:

$$
\begin{aligned}
& K_{x 1}=\left[\begin{array}{llllll}
-0.0417 & 0.0003 & -0.0781 & 0 & -6.0115 & 0.3879
\end{array}\right] \times 10^{6} \\
& K_{d 1}=[-210.4250]
\end{aligned}
$$

And the tie line power between both control areas is chosen as:

$$
H_{12}=\left[\begin{array}{cccccc}
0 & 0 & 0 & 0 & 0 & 0.0327 \\
0 & 0 & 0 & 0 & 0 & 0 \\
0 & 0 & 0 & 0 & 0 & 0 \\
0 & 0 & 0 & 0 & 0 & -0.0054 \\
0 & 0 & 0 & 0 & 0 & 0 \\
0 & 0 & 0 & 0 & 0 & 0
\end{array}\right]
$$

where:

$A_{1}=A_{2}, B_{1}=B_{2}, \Gamma_{1}=\Gamma_{2}, K_{x 1}=K_{x 2}$ and $K_{d 2}=K_{d 2}$

We simulate and illustrate the response of the two-area power system with nominal parameters in Table 1 and the extended observer is tested by applying the load disturbance of $\Delta P_{d 1}=0.01$ (p.u, MW) at $t=1 \mathrm{~s}$ and at $\Delta P_{d 2}=0.015$ (p.u, MW) $t=1 \mathrm{~s}$. The simulation results of the two-area multi-area power network for case 1 that the proposed GESO are presented in Fig. 5 to Fig. 8. It is simply to observe in Fig. 5 that the frequency variation converges to zero in about $2 \mathrm{~s}$. Fig. 6 and Fig. 8 indicate the mechanical power deviation and control signal deviation of two control areas. Fig. 7 presents that the tie-line power deviations reach to zero with the designed controller. In comparison between the results achieved by using proposed control method with previous research in [5-6], [15], the results of using the proposed controller are to reduce the setting time and overshoots of both in power networks. So, it is proved that the proposed controller is powerful and effective.

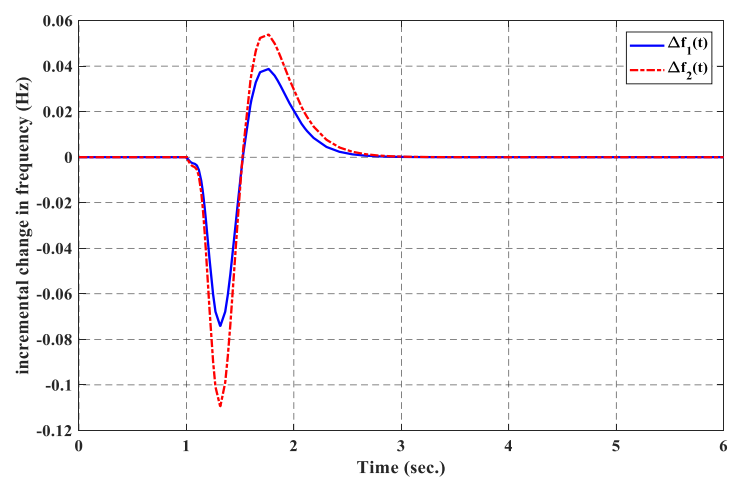

Fig. 5: Frequency deviation of two control areas.

Case 2. In the second case, the performance of proposed GESO scheme is in the presence of nonlinear term such as matched uncertainties to constate the model of the system in Fig. 1 and Fig. 3. 
To combine between system matrix of hydro power plant and parameter values in Table 1 , the matrix values of the power system are calculated as:

$$
\begin{gathered}
A_{1}=\left[\begin{array}{cccccc}
-0.05 & 6 & 0 & 0 & 0 & -0.0327 \\
0.4831 & -6.6667 & 6.7362 & 1.0899 & 0 & 0 \\
-0.1697 & 0 & -0.0348 & -3.2986 & 0 & 0 \\
-1.3889 & 0 & 0 & -3.3333 & 0 & 0 \\
0.4250 & 0 & 0 & 0 & 0 & 0.0054 \\
6.2832 & 0 & 0 & 0 & 0 & 0
\end{array}\right] \\
B_{1}=\left[\begin{array}{lllllll}
0 & -1.1594 & 0.5797 & 3.3333 & 0 & 0
\end{array}\right]^{T} \\
\Gamma_{1}=\left[\begin{array}{llllll}
-6 & 0 & 0 & 0 & 0 & 0
\end{array}\right]^{T}
\end{gathered}
$$

Feedback control gain in this case can be designed as:

$$
\begin{aligned}
& K_{x 1}=\left[\begin{array}{llllll}
-0.0084 & -0.0012 & 0.0043 & -0.0011 & -6.8195 & 0.4481
\end{array}\right] \times 10^{6} \\
& K_{d 1}=[-210.4250]
\end{aligned}
$$

And the tie-line power between both control areas are chosen as:

$$
H_{12}=\left[\begin{array}{cccccc}
0 & 0 & 0 & 0 & 0 & 0.0327 \\
0 & 0 & 0 & 0 & 0 & 0 \\
0 & 0 & 0 & 0 & 0 & 0 \\
0 & 0 & 0 & 0 & 0 & -0.0054 \\
0 & 0 & 0 & 0 & 0 & 0 \\
0 & 0 & 0 & 0 & 0 & 0
\end{array}\right]
$$

where:

$A_{1}=A_{2}, B_{1}=B_{2}, \Gamma_{1}=\Gamma_{2}, K_{x 1}=K_{x 2}$ and $K_{d 2}=K_{d 2}$.

We change the hydro power plant with hydraulic governor instead of thermal power plant and the step load disturbances are kept the same with first case. The deviations in frequency of first and second power area are shown in Fig. 9. Fig. 10 shows mechanical power deviation of two control areas. Fig. 11 and Fig. 12 display in order the tie-line power deviation and control signal deviation. In each control area, the closed loop responses applying the GESO controller are simply to observe from Fig. 9 to Fig. 12 that the response performance is better in terms of settling time about $1 \mathrm{~s}$ and under/overshoots, in comparison to the recent others proposed in [5$6],[15]$.

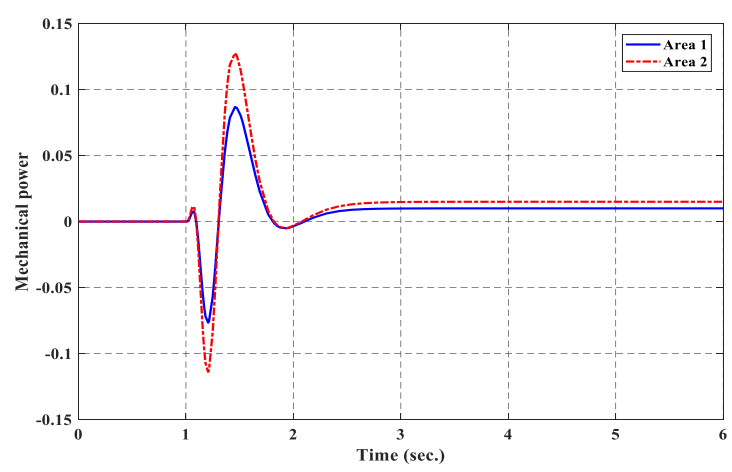

Fig. 6: Mechanical power deviation.

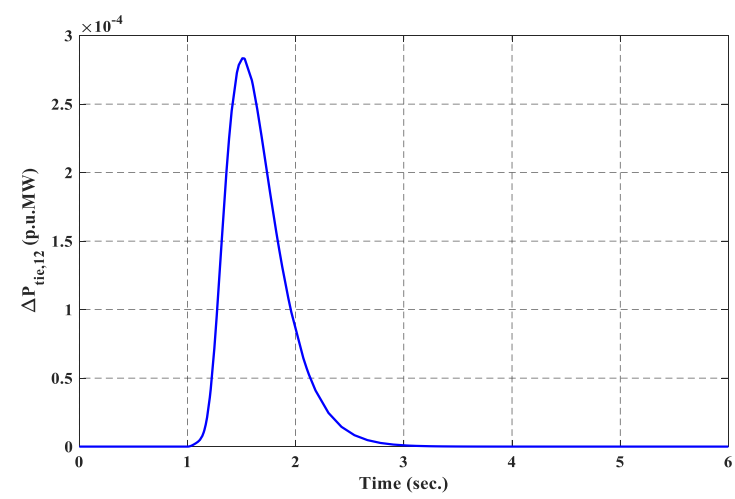

Fig. 7: The tie-line power deviation. 


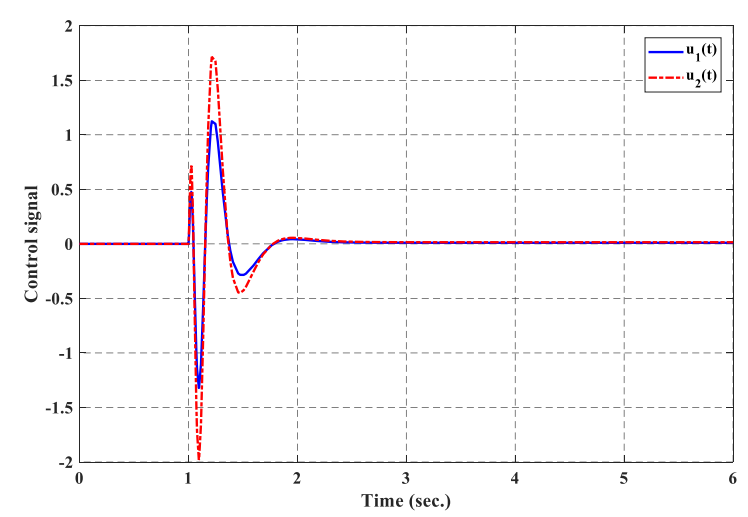

Fig. 8: Control signal deviation of two control areas.

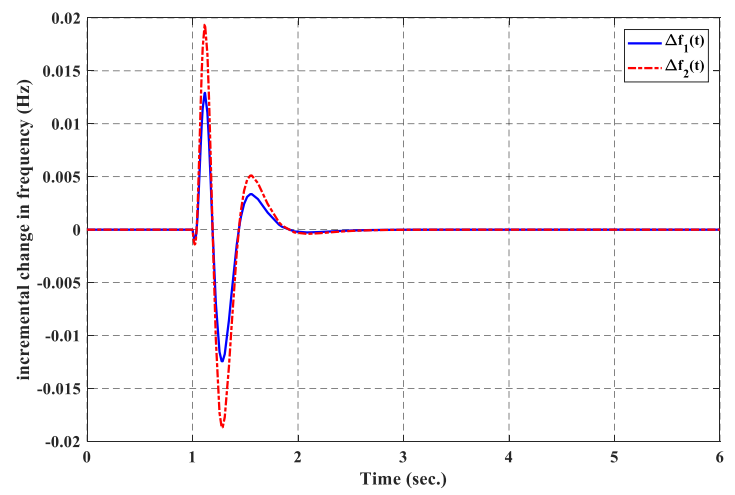

Fig. 9: Frequency deviation of two control areas.

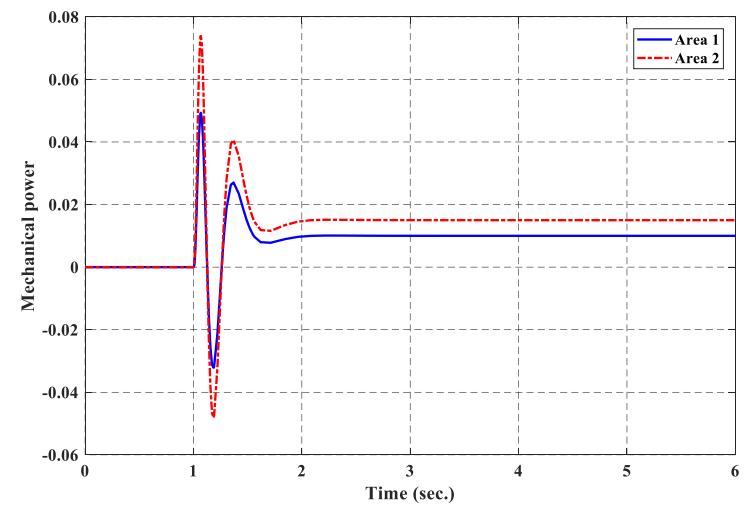

Fig. 10: Mechanical power deviation.

Remark 3: From the reporting of simulation in case 1 and case 2, the proposed approach is one of main objectives to finalize the matched disturbances and achieve shorter setting time and smaller transient deviation in terms of load disturbances for interconnected power system by

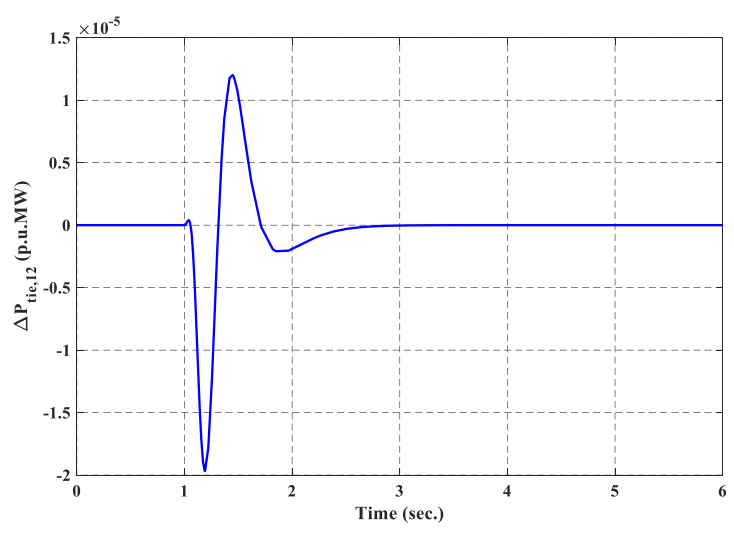

Fig. 11: The tie-line power deviation.

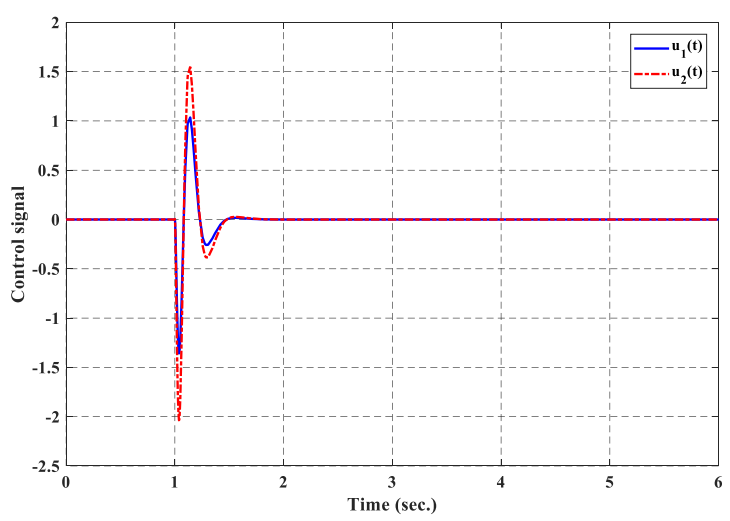

Fig. 12: Control signal deviation.

applying of the proposed GESO law. So, some limitations of other schemes in recent papers [5$6]$ and [15] have been resolved.

Case 3. Now, the suggested GESO control approach is used to examine by comparing with traditional LFC [5-6], [15] at random load variations. In this specific case, we consider the power system which includes two kinds of the plant as thermal power plant with reheat turbine and hydro power plant with mechanical hydraulic governor. The parameter values of the complex power system are calculated given as: 
In the first area:

$$
\begin{gathered}
A_{1}=\left[\begin{array}{cccccc}
-0.05 & 6 & 0 & 0 & 0 & -0.0327 \\
0 & -0.1 & -15667 & 1,6667 & 0 & 0 \\
0 & -3.3333 & 3.3333 & 0 & 0 & 0 \\
-5.2083 & 0 & 0 & -12.5 & 0 & 0 \\
0.425 & 0 & 0 & 0 & 0 & 0.0054 \\
6.2832 & 0 & 0 & 0 & 0 & 0
\end{array}\right] \\
B_{1}=\left[\begin{array}{lllllc}
0 & 0 & 0 & 12.5 & 0 & 0
\end{array}\right]^{T} \\
\Gamma_{1}=\left[\begin{array}{llllll}
-6 & 0 & 0 & 0 & 0 & 0
\end{array}\right]^{T}
\end{gathered}
$$

The feedback control gain can be designed:

$$
\begin{aligned}
& K_{x 1}=\left[\begin{array}{llllll}
-0.0417 & 0.0003 & -0.0781 & 0 & -6.0115 & 0.3879
\end{array}\right] \times 10^{6} \\
& K_{d 1}=[-210.4250]
\end{aligned}
$$

And the second area:

$$
\begin{gathered}
A_{2}=\left[\begin{array}{cccccc}
-0.05 & 6 & 0 & 0 & 0 & -0.0327 \\
0.4831 & -6.6667 & 6.7362 & 1.0899 & 0 & 0 \\
-0.1697 & 0 & -0.0348 & -3.2986 & 0 & 0 \\
-1.3889 & 0 & 0 & -3.3333 & 0 & 0 \\
0.4250 & 0 & 0 & 0 & 0 & 0.0054 \\
6.2832 & 0 & 0 & 0 & 0 & 0
\end{array}\right] \\
B_{2}=\left[\begin{array}{llllllll}
0 & -1.1594 & 0.5797 & 3.3333 & 0 & 0
\end{array}\right]^{T} \\
\Gamma_{2}=\left[\begin{array}{lllllll}
-6 & 0 & 0 & 0 & 0
\end{array}\right]^{T}
\end{gathered}
$$

The feedback control gain can be designed:

$$
\begin{aligned}
& K_{x 2}=\left[\begin{array}{llllll}
-0.0084 & -0.0012 & 0.0043 & -0.0011 & -6.8195 & 0.4481
\end{array}\right] \times 10^{6} \\
& K_{d 2}=[-210.4250]
\end{aligned}
$$

And the tie-line power between two-area are chosen as:

$$
H_{12}=\left[\begin{array}{cccccc}
0 & 0 & 0 & 0 & 0 & 0.0327 \\
0 & 0 & 0 & 0 & 0 & 0 \\
0 & 0 & 0 & 0 & 0 & 0 \\
0 & 0 & 0 & 0 & 0 & -0.0054 \\
0 & 0 & 0 & 0 & 0 & 0 \\
0 & 0 & 0 & 0 & 0 & 0
\end{array}\right]
$$

The actual random load disturbances are implemented and applied in both control areas of power system as presented in Fig. 13 to Fig. 17. In flowing detail, Fig. 13 shows the load variations of two control areas. The deviation in frequency of both areas is shown in Fig. 14.
Fig. 15, Fig. 16 and Fig. 17 plot the frequency deviation, mechanical power deviation and control signal deviation of two control areas. The generalized extended observer controller is still designed to compute and estimate system variables. In comparison between the deviations in frequency from [5-6], [15] and the simulation results of the proposed GESO controller, the significant improvement is to reduce the magnitude of oscillation as well as minimize under or overshoots and settling time in the response performance.

Remark 4: To observe of 3 cases of simulation results above, the proposed GESO ap- 


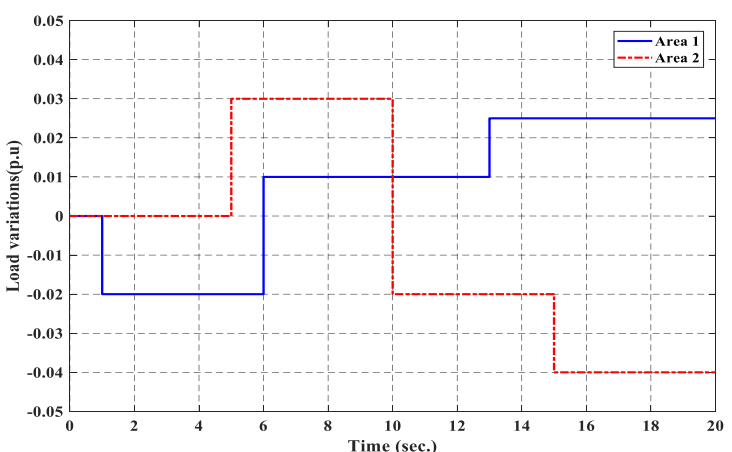

Fig. 13: Load variations of two control areas.

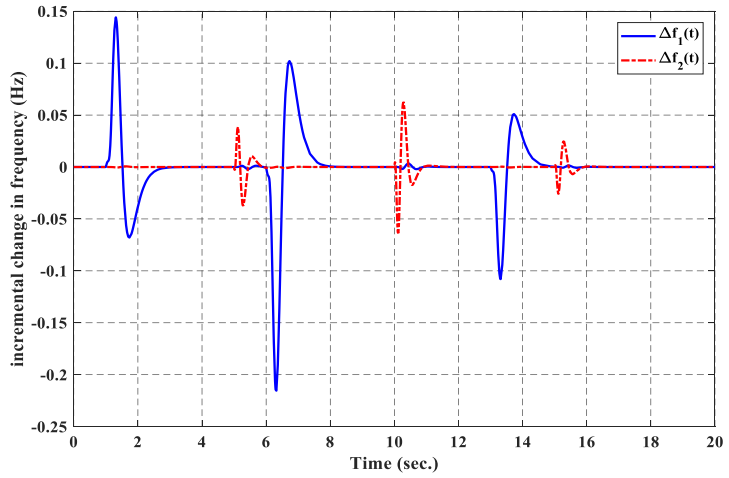

Fig. 14: Frequency deviation of two control areas.

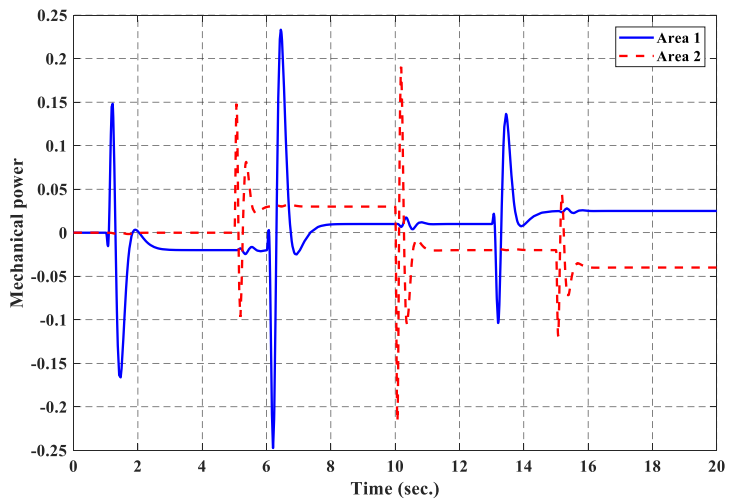

Fig. 15: Mechanical power deviation.

proach achieves affectively the response performance under conditions such as the matched uncertainties and random load variations appearing in complex power networks. The suggested control scheme is applied and developed to eliminate load disturbances and to restore the nominal point of system performance, and to reduce the influence of external disturbances.

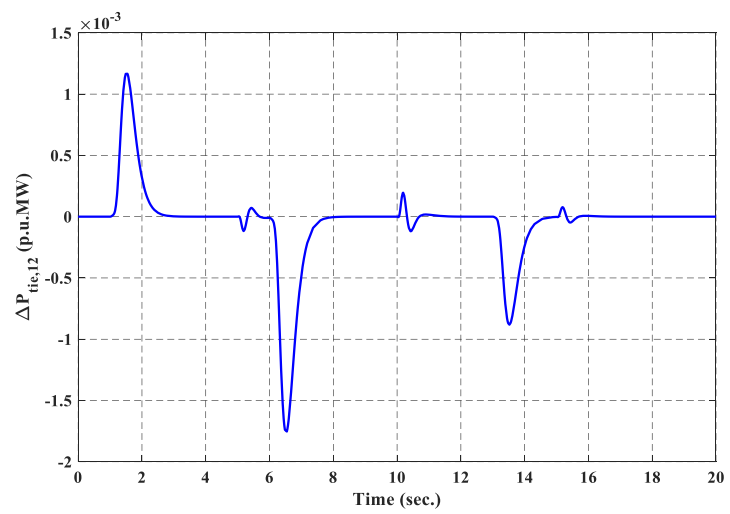

Fig. 16: The tie-line power deviation.

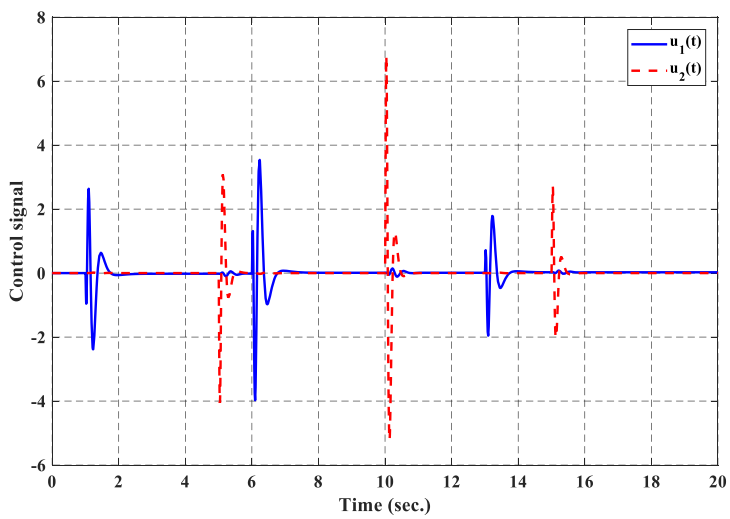

Fig. 17: Control signal deviation of two control areas.

Case 4. In this part, the frequency variation, tie-line power and control input signal are presented from Fig. 18 to Fig. 20 at different step load disturbances $\Delta P_{d 1}=0.1$ (p.u.MW) at $t=0 \mathrm{~s}$ in the first-area, $\Delta P_{d 2}=0.05$ (p.u.MW) at $t=0 \mathrm{~s}$ in the second-area. The non-reheat turbine is applied to both areas and system parameters are used the same with the one given in [28].

Delve into data analysis, the goal of any load frequency controllers is to return frequency value to the safe point. It is to be clear that we observe in Fig. 18 and Fig. 19, the proposed GESO controller obtains the normal range in frequency about $1 \mathrm{~s}$ at both areas and decreases tie-line power variation to zero about $3 \mathrm{~s}$, respectively. The proposed GESO controller also reduces and minimizes overshoot and settling time as compared with recent studies in [5-6], [15] and with observer controller in [28]. 


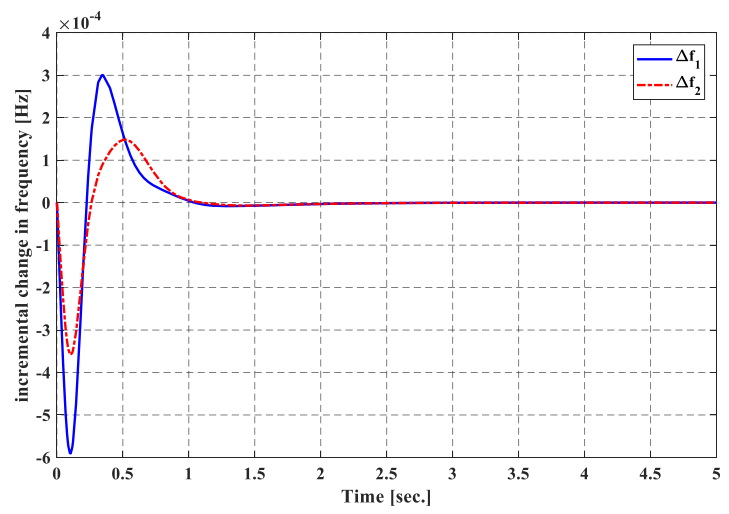

Fig. 18: Frequency deviation of two control areas.

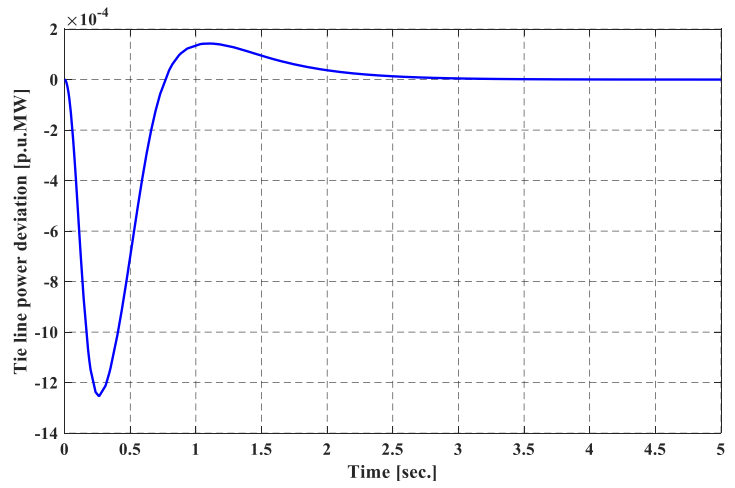

Fig. 19: The tie-line power variation.

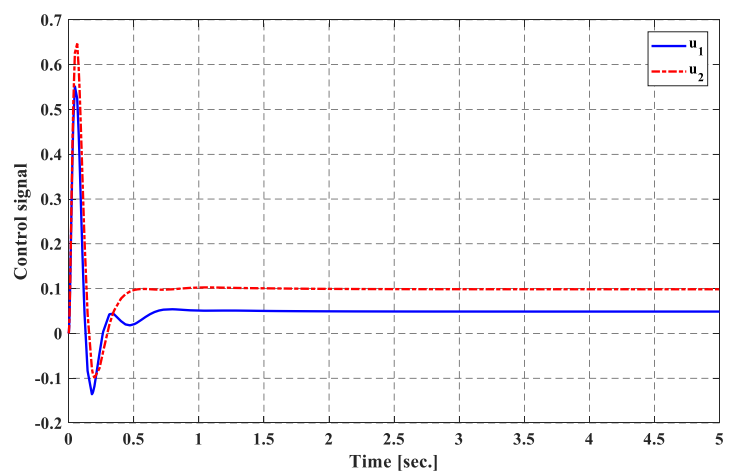

Fig. 20: Control signal.

Remark 5. It is to be noted that the suggested GESO approach has ability to estimate and compensate exactly under the matched uncertainty. In particular, the proposed control scheme makes better the system damping characteristic.
Simulation 2. In the last case, we consider the dynamic models utilized for simulation of physical constraints of GDB and GRC in the thermal power plant with reheat turbine and the hydro power plant with mechanical hydraulic governor in Fig. 21.

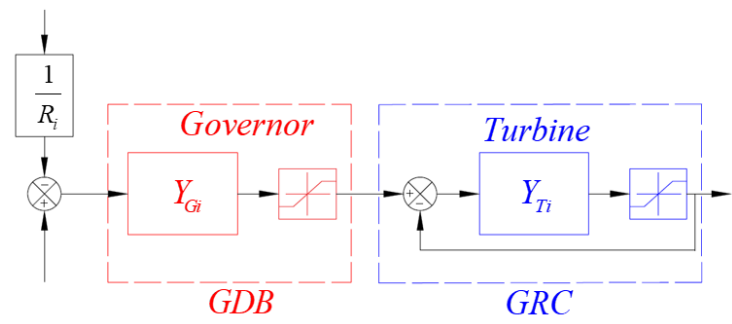

Fig. 21: Nonlinear model with GDB and GRC [32].

We test the proposed controller with the step load disturbance of $\Delta P_{d 1}=0.01$ (p.u MW) at $t=1 \mathrm{~s}$ and $\Delta P_{d 2}=0.03$ (p.u MW) at $t=1 \mathrm{~s}$. Fig. 22, Fig. 23 and Fig. 24 represent the frequency variation, tie-line power variation and mechanical power variation in each control area. The control signal of both control area illustrates in Fig. 25. As it is clear, with the proposed GESO controllers, the transient oscillations are determined a longer time with larger amplitude than in the cases of without considering the GRC and GDB in case 1, case 2, case 3 in simulation 1 . The proposed control strategy has also discovered satisfactory even in presence of GRC, GDB and step load disturbances in comparison with [30]. The overshoot percentage and settling time are synchronously significantly decreased in the transient performance of the suggested GESO controller.

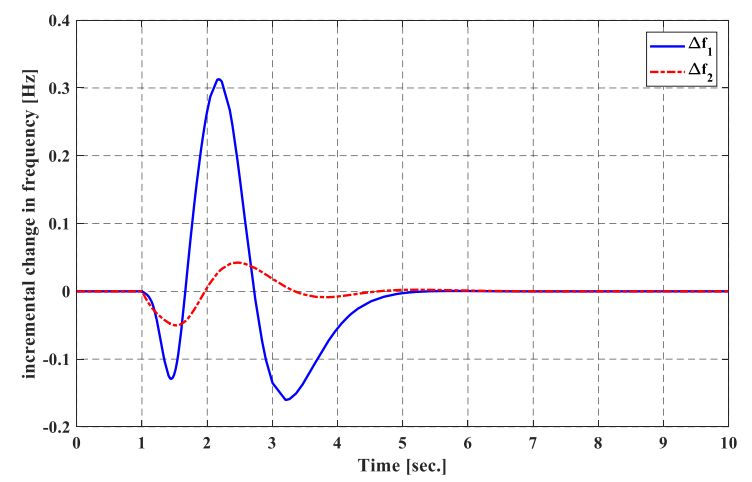

Fig. 22: Frequency variation of two control areas. 


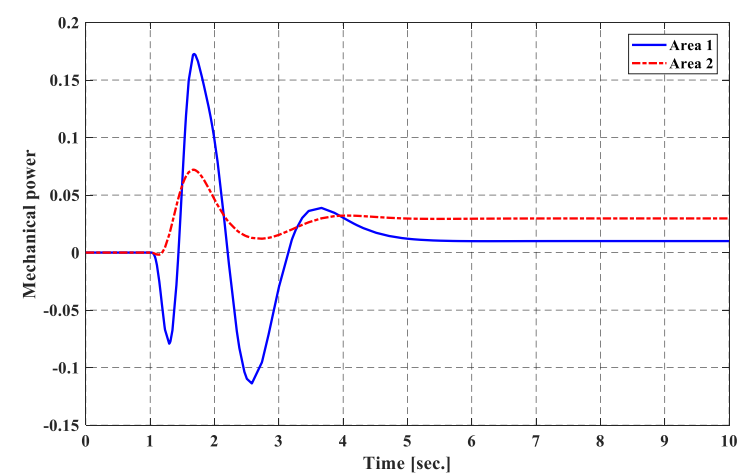

Fig. 23: Mechanical power deviation.

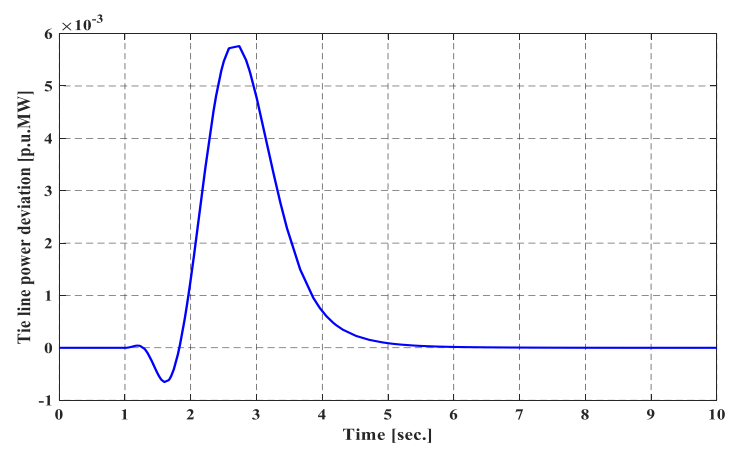

Fig. 24: The tie-line power deviation.

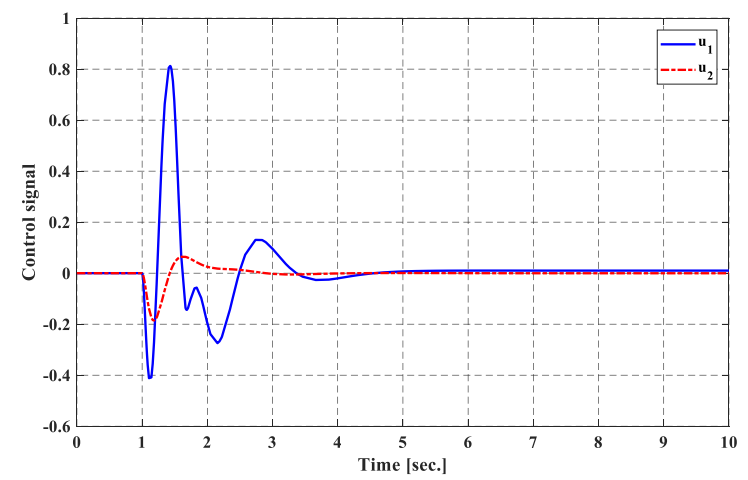

Fig. 25: Control signal.

Remark 6: The GRC and GDB impact significantly to feedback signal of the interconnected power network. To show the robustness of the proposed GESO, the simulation results are used to compare with the case of considering in [30] or without considering the GDB and GRC nonlinearity effects in [31]. The proposed controller clearly indicates that transient performance has adapted with required condition such as the setting time and under/overshoot in comparison with previous research. Thus, the small deviations in frequency with the proposed GESO have less effect on the plant reserve capacity and power market.

\section{Conclusions}

To solve the problem of unmeasurable of system states in interconnected power system due to lack of sensor, the load frequency control based generalized extended state observer is proposed in this paper. The generalized extended state observer is used to estimate the unmeasurable of system states and load disturbances. The proposed scheme of making the interconnected power system is not only secure and stable but also useful to solve the satisfactory performance with system parameter uncertainties. The simulation results point out that the LFC based GESO approach improves the system dynamic response to fast response in setting time and to reduce over or undershoots in power network with the dynamic model of thermal power plant with reheat turbine and hydro power plant. Moreover, the report of simulation results is used to compare with the cases of considering and without considering the GDB and GRC nonlinearity effects on power network. It is evident that the robustness of the suggested controller in terms of stability and effectiveness of system.

\section{Acknowledgements}

This research was funded by Science and Technology Development of Ton Duc Thang University (FOSTECT), website: http://fostect.tdtu.edu.vn, under Grant FOSTECT.2017.BR.05

\section{References}

[1] Chaturvedi, D.K. (2008). Techniques and its Applications in Electrical Engineering Springer. 
[2] Vittal, V., Mc Calley, J.D., Anderson, P.M., \& Fouad, A.A. (2019). Wiley 3rd Edition, Power System Control and Stability.

[3] Fu, C., \& Tan, W. (2018). Decentralized Load Frequency Control for Power Systems with Communication Delays via Active Disturbance Rejection. IET Generation, Transmission 83 Distribution, 12(6), 1751-8687.

[4] Bernard, M.Z., Mohamed, T.H., Qudaih, Y.S., \& Mitani, Y. (2014). Decentralized load frequency control in an interconnected power system using Coefficient Diagram Method. International Journal of Electrical Power \& Energy Systems, 63(5), 65-172.

[5] Parmar, K.P.S., Majhi, S., \& Kothari, D.P. (2012). Load frequency control of a realistic power system with multi-source power generation. International Journal of Electrical Power 83 Energy Systems, 42(1), 426-433.

[6] Parmar, K.P.S., Majhi, S., \& Kothari, D.P. (2014). LFC of an interconnected power system with multi-source power generation in deregulated power environment. International Journal of Electrical Power 83 Energy Systems, 57(2), 277-286.

[7] Yousef, H.A., AL-Kharusi, K., Albadi, M.H., \& Hosseinzadeh, N. (2014). Load Frequency Control of a Multi-Area Power System. An Adaptive Fuzzy Logic Approach, IEEE Transactions on Power Systems, 29(4), 1822-1830.

[8] Zeng, G.-Q., Xie, X.-Q., \& Chen, M.-R., (2017). An Adaptive Model Predictive Load Frequency Control Method for Multi-Area Interconnected Power Systems with Photovoltaic Generations. Electrical Power and Energy System, 10(11), 1840.

[9] Rehiara, B.A., Yorino, N., Sasaki, Y., \& Zoka, Y. (2020). An Adaptive Load Frequency Control Based on Least Square Method. Advances in Modelling and Control of Wind and Hydrogenators, 49(3), 220 .
[10] Dahab, Y.A., Abubakr, H., \& Mohamed, T.H. (2020). Adaptive Load Frequency Control of Power Systems using ElectroSearch Optimization Supported by the Balloon Effect. IEEE Access, 7408-7422.

[11] Anwar, M.N.\& Pan, S., (2015). A New PID Load Frequency Controller Design Method in Frequency Domain Through Direct Synthesis Approach. Electric Power and Energy Systems, 67(4), 560-569.

[12] Kouba, N., Menaa, M., Hasni, M., et al. (2015). Load frequency control in multiarea power system based on fuzzy logic-PID controller. IEEE Int. Conf. on Smart Energy Grid Engineering SEGE, Oshawa, Canada, 15(1), 1-6.

[13] Farahani, M., Ganjefar, S., Alizadeh, M. (2012). PID controller adjustment using chaotic optimization algorithm for multiarea load frequency control. IET Control Theory Appl., 6(2), 1984-1992.

[14] Yang, M., Yang, F., Chengshan, W., \& Peng, W. (2013). Decentralized Sliding Mode Load Frequency Control for MultiArea Power Systems. IEEE Transactions on Power System, 28(4), 4301-4309.

[15] Guo, J. (2020). Application of A Novel Adaptive Sliding Mode Control Method to the Load Frequency Control. European Journal of Control, 5(2), 3580-3601.

[16] Mu, C., Tang, Y., \& He, H., (2017). Improved Sliding Mode Design for Load Frequency Control of Power System Integrated an Adaptive Learning Strategy. IEEE Transactions on Industrial Electronics, 64(8), 6742-6751.

[17] Trip, S., Cucuzzella, M., De Persis, C., van der Schaft, A., \& Ferrara, A. (2019). Passivity-Based Design of Sliding Modes for Optimal Load Frequency Control. IEEE Transactions on Control Systems Technology, 27(5), 1893-1906.

[18] Li, H.Y., Shi, P., Yao, D.Y., \& Wu, L.G. (2016). Observer-Based Adaptive Sliding Mode Control of Nonlinear Markovian 
Jump Systems. Automatica, 64(1), 133142 .

[19] Khayati, K., (2015). Multivariable Adaptive Sliding-Mode Observer-Based Control for Mechanical Systems. Canadian Journal of Electrical and Computer Engineering, 38(3), 253-265.

[20] Wang, B., Shi, P., Karimi, H.R., \& Lim, C.C. (2013). Observer-Based Sliding Mode Control for Stabilization of a Dynamic System with Delayed Output Feedback. Mathematical Problems in Engineering, 3(1), 1-6.

[21] Yang, B., Yu, T., Shu, H., Yao, W., \& Jiang, L. (2018). Sliding-Mode Perturbation Observer-Based Sliding-Mode Control Design for Stability Enhancement of MultiMachine Power Systems. Transactions of the Institute of Measurement and Control, 41(5), 1418-1434.

[22] Mi, Y., Fu, Y., Li, D., Wang, C., Loh, P.C., \& Wang, P. (2016). The Sliding Mode Load Frequency Control For Hybrid Power System Based on Disturbance Observer. International Journal of Electrical Power \& Energy Systems, 74 (1), 446-452.

[23] Pawar, S.N., Chile, R.H., \& Patre, B.M. (2017). Design of Generalized Extended State Observer based Control for Nonlinear Systems with Matched and Mismatched Uncertainties. Indian Control Conference (ICC), 4-6.

[24] Yao, J., Jiao, Z., \& Ma, D. (2014). Adaptive Robust Control of DC Motors with Extended State Observer. IEEE Transactions on Industrial Electronics, 61(7), 3630-3637.

[25] Wang, S., Ren, X., Na, J., \& Zeng, T. (2017) Extended-State-Observer-Based Funnel Control for Nonlinear Servomechanisms with Prescribed Tracking Performance. IEEE Transactions on Automation Science and Engineering, 14 (1), 98-108.

[26] Hossain, M., \& Peng, C. (2020). Load Frequency Control for multiarea power systems under DoS attacks. Information Sciences, 243(1), 437-453.
[27] Haes, A., Hamedani, H., Mohamad, G., Hatziargyriou, E., \& Nikos, D. (2019). A Decentralized Functional Observer based Optimal LFC Considering Unknown Inputs, Uncertainties and Cyber-Attacks. IEEE Transactions on Power Systems, $34(6), 4408-4417$.

[28] Chen, C., Zhang, K., Yuan, K., \& Wang, W. (2017). Extended Partial States Observer Based Load Frequency Control Scheme Design for Multi-area Power System Considering Wind Energy Integration. IFAC-Papers On-Line, 50(1), 4388-4393.

[29] Rinaldi, G., Cucuzzella, M., \& Ferrara, A. (2017). Third order sliding mode observerbased approach for distributed optimal load frequency control. IEEE Control Systems Letters, 1(2), 215-220.

[30] Prasad, S., Purwar, S., \& Kishor, N. (2019). Load frequency regulation using observer based non-linear sliding mode control. International Journal of Electrical Power $\mathcal{G}$ Energy Systems, 108(1), 178-193.

[31] Prasad, S. (2020). Counteractive control against cyber-attack uncertainties on frequency regulation in the power system: IET Cyber-Physical Systems. Theory \&6 Applications Research, 5(4), 394-408.

[32] Bevrani, H. (2014). Robust Power System Frequency Control, Power Electronics and Power Systems, Springer.

\section{About Authors}

Anh-Tuan TRAN received B.Eng. degree in electrical engineering, Ton Duc Thang University (TDTU), Ho Chi Minh City, Vietnam. Currently, he is M.Eng. student in Electrical Engineering, TDTU, HCMC, Vietnam. His research topics included load frequency control and automatic voltage regulator to power systems with sliding mode control and optimal control. 
Phong Thanh TRAN received the B.Eng. degree in physics and electronics with a major in automation and control at Ho Chi Minh University of Science, Ho Chi Minh, Vietnam, in 2010, and the M.Eng. degree in electrical engineering with a major in automation and control at International University, Ho Chi Minh National University, in 2015. His current research activities include power systems, siding mode control, dynamic system and load frequency control.
Van Van HUYNH has completed the Ph.D degree in automation and control from Da-Yeh University, Taiwan. He is currently a Lecturer in the Faculty of Electrical and Electronics Engineering, Ton Duc Thang University, Ho Chi Minh City, Vietnam. He has published totally 10 journal papers and more than 11 international conference papers. His current research interests are in sliding mode control, variable structure control, and power system control. 